\title{
NANOMETER GAPS BY FEEDBACK-CONTROLLED ELECTROMIGRATION
}

\author{
Victor Chi-Yuan Shih, Siyang Zheng, Alan Chang and Yu-Chong Tai \\ Electrical Engineering, 136-93, California Institute of Technology \\ Pasadena. CA 91125, USA, cyshih@caltech.edu
}

\begin{abstract}
Nanometer-sized gap (or nanogap) is one of the most fundamental devices in the nanotechnology field. Park et. al., [1] first proposed the open-circuit electromigration method to fabricate nanogaps, but the process is only repeatable if $\mathrm{Au}$ film is thinner than $20 \mathrm{~nm}$. To overcome these drawbacks, we develop the feedback-controlled electromigration process and find that not only repeatable nanogaps can be created in thicker film (up to $120 \mathrm{~nm}$ or thicker in our experiments), but superior gap size control and topology are obtained. Moreover, we develop two new approaches to make free-standing nanogaps. The tunneling current between the nanogap electrodes was used to demonstrate a sensitive pressure and/or temperature sensor. Finally, we also develop a simple thermal-expansion method to measure the gap size without needing delicate instrument.
\end{abstract}

\section{INTRODUCTION}

There have been growing interests in nanometer gap applications. To make nanogaps, techniques using electromigration [1], mechanical break junction [2], chemical electroplating [3] and e-beam lithography [4] have been reported. The mechanical break junction method requires a delicate forcing system to generate and maintain the gaps. The e-beam lithography is quite direct but it is expensive and it is difficult to go below certain feature size (typically $10 \mathrm{~nm}$ ) depending on the thickness of the target film [4]. The electroplating method, while relatively simple in system setup, needs precise feedback control and the needed electrolyte can cause contamination issues. Park et. al., [1] first proposed the even simpler open-circuit electromigration method to fabricate nanogaps, but the process is only repeatable if $\mathrm{Au}$ film is thinner than $20 \mathrm{~nm}$. In this paper, we further develop a "feedback-controlled" electromigration process to extend the capability of Park's method. This modified approach now provides a convenient way to generate nanogaps in gold films up to $120 \mathrm{~nm}$. Nanogap formation in thicker film is currently under study.

\section{DESIGN AND FABRICATION}

The gold film chip used for nanogap formation experiments is shown in Fig. 1 , in which a $1-\mu$ m-wide neck of gold lead is the key design. The fabrication process for the chip begins with growing a $200 \mathrm{~nm}$ thermal oxide on a single crystal silicon substrate. Then a $500 \mathrm{~nm}$ amorphous

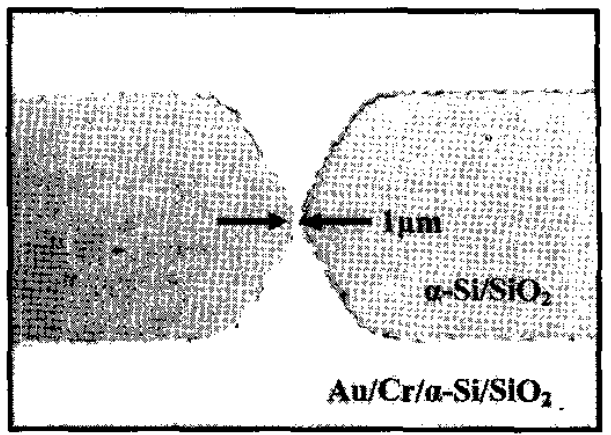

Fig. 1 Au bridge structure patterned with wet etching. $\mathrm{Cr}$ layer is carefully timed-etched to avoid undercut.

silicon is sputtered on the oxide layer. . Next, a $\mathrm{Cr} / \mathrm{Au}$ $(5 / 120 \mathrm{~nm})$ layer is thermally evaporated and patterned to define the bridge area with the neck. Here, the $\mathrm{Cr}$ under the $\mathrm{Au}$ is purposely remained so as to provide good heat conduction during electromigration between $\mathrm{Au}$ and the substrate.

After gold patterning and photoresist stripping, the chip is dipped into Au etchant for a few seconds. Fig. 2 then shows the SEM pictures, which clearly reveal the grain structures of Au film. It is experimentally found that these grain structures determine the position of nanogap formation

\section{FEEDBACK-CONTROLLED ELECTROMIGRATION}

To generate electromigration on $\mathrm{Au}$ film, the current density in Au needs to be $>10^{8} \mathrm{~A} / \mathrm{cm}^{2} \cdot[5]$. When a current is applied to our design structure, the current density in the neck region is much larger than the other part. The Au dipping etches faster at the grain boundaries so that the current

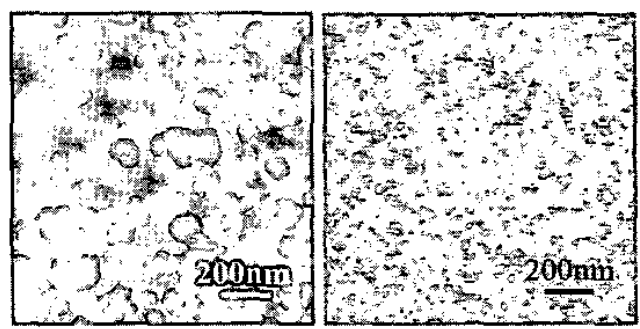

Fig. 2 SEM pictures showing Au surface with (left) and without (right) dipping procedure in Au etchant. Dip-etching clearly reveals grain structure.

TRANSDUCERS ' 03

The 12th International Conference on Solid State Sensors, Actuators and Microsystems, Boston, June 8-12, 2003 


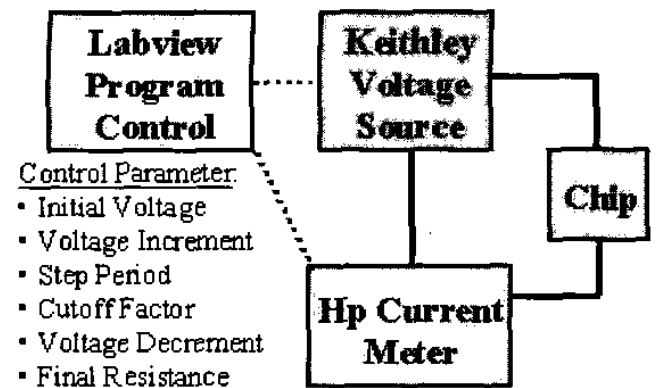

Fig. 3 System setup for electromigration process.

density is also higher there. Finally, the Au atom mobility at the grain boundary is also higher[6]. These arguments conclude that electromigration occurs more easily at the grain boundaries than inside the grains. As a whole, our sample should produce the nanogap right at the neck and along some grain boundary through the process of electromigration.

In our experiments, a feedback-control mechanism is then used to apply a minimum current through the bridge just to generate significant electromigration in the neck region. Fig. 3 shows the system setup. Labview is used to close the feedback loop between the voltage source and the current meter. Experimentally, we found several important precautions. First, human bodies should be electrically grounded to prevent electrostatic discharging to the system during operation. From experiences, electric spikes can often result in a big current flow through the chip and melt the bridges immediately. Probe contacts should be made right outside the gold bridge region to make sure that most of the voltage drop is applied across the bridge. Once the control program starts, the voltage source will output an initial voltage $\left(\mathrm{V}_{\mathrm{i}} \sim 0.3 \mathrm{~V}\right)$. After each period $(\mathrm{T} \sim 50 \mathrm{sec})$, the output voltage increases by $V_{\text {inc }}(\sim 30 \mathrm{mV})$. When the voltage reaches a threshold value, some local electromigration will initiate and start generating a gap that will propagate across the width (Fig. 4). This gap propagation results in increasing resistance and decreasing current (Fig. 5). In our algorithm, when the current decreases to a preset cutoff factor (CF), the output voltage will be reduced by $\mathrm{V}_{\mathrm{dec}}$ to avoid uncontrolled local melting. The program stops when the bridge resistance reaches a target value $\left(R_{f}\right)$ and the gap formation is complete. Very importantly, the $\mathrm{Cr}$ layer under $\mathrm{Au}$ serves as an alternative current path when the nanogap in Au layer is complete ( $\mathrm{Cr}$ has a higher resistance to electromigration [7]). It helps to keep the voltage drop across the nanogap

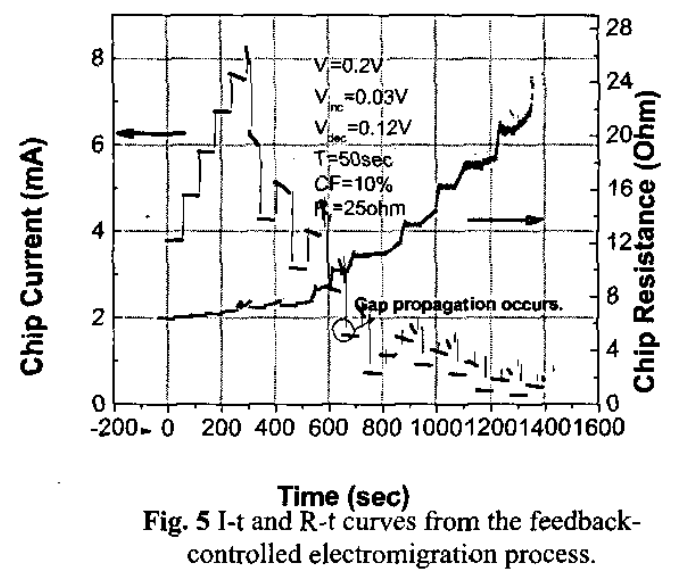

small through out the process. After the nanogap is formed, we remove chrome in the gap region by wet etching. After the $\mathrm{Cr}$ removal, the created nanogap has a resistance ranging from $M \Omega$ 's to G $\Omega$ 's, which indicates the gap completion. The finished nanogaps are then examined by SEM to determine gap geometries.

In general, this feedback-controlled electromigration approach has a yield higher than $50 \%$ in making nanogap size of $10 \mathrm{~nm}$ or smaller. However, due to the fact that electromigration is a destructive process, it is not possible to get repeatable gap geometries due to irregular grain boundaries [8].

In our study, gaps generated on amorphous silicon layer with or without the feedback mechanism are compared (shown in Figs. 6a\&b). Moreover, suspended gaps, which are formed after the amorphous silicon is removed before electromigration, are also investigated (shown in Figs. $6 c \& d)$. The no-feedback-control experiment is carried out by ramping up the applied voltage to the bridge at a constant slew rate $(\sim 50 \mathrm{mV} / \mathrm{sec})$ and stopping the voltage output when bridge resistance suddenly increases. Results clearly show that the feedback-controlled electromigration reduces local gold melting by passing only minimum required current to the bridge and therefore results in smaller gap sizes.

In order to measure tunneling current through the gaps, we have built nanogaps directly on top of thermal oxide to avoid any substrate conduction. In cases of nanogaps smaller than $5 \mathrm{~nm}$, tunneling currents can be measured as

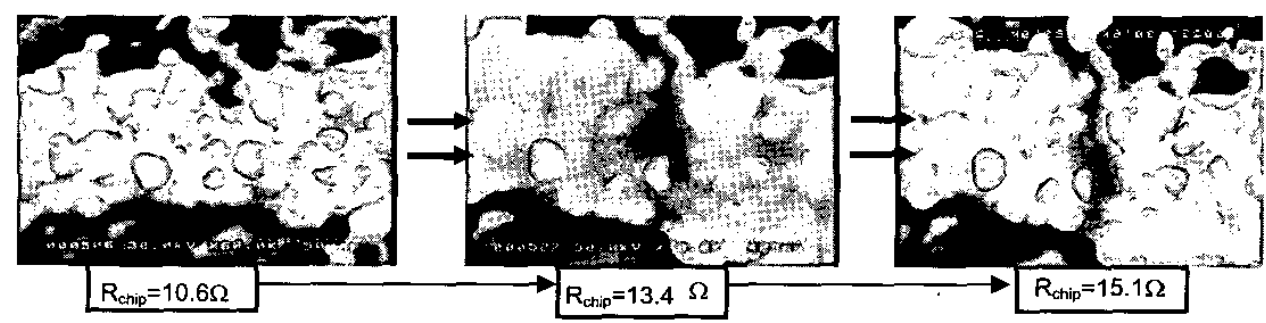

Fig. 4 SEM shows gap propagation process on one bridge during a feedback-controlled electromigration.

TRANSDUCERS ' 03

The 12th International Conference on Solid State Sensors, Actuators and Microsystems, Boston, June 8-12, 2003 


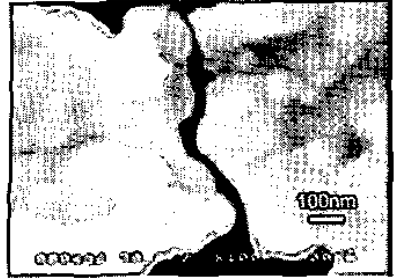

(a)

(c)

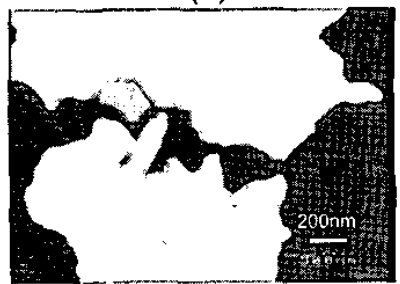

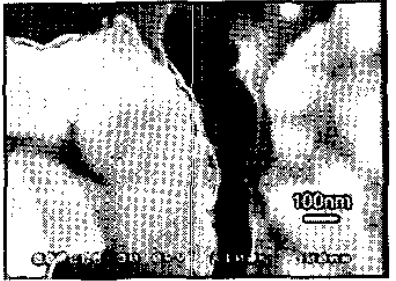

(b)

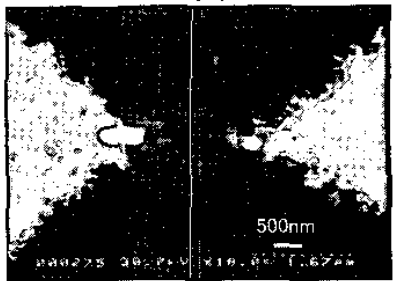

(d)
Fig. 6 SEM pictures of (a) nanogaps on $\alpha$-Si substrate generated with feedback control, (b) without feedback control. (c) Suspended gaps generated with feedback control, (d) without feedback control.

shown in Fig. 7. Based on $[9,10]$, no field emission and gas ionization should happen with the electric fields (i.e., voltages) used in our I-V measurements. The current as measured, therefore, should be the direct tunneling current. Since the direct tunneling is measurable only if gap size is $<5 \mathrm{~nm}$ [11], we conclude that the feedback-controlled electromigration process can produce sub-5nm gaps.

\section{FREESTANDING NANOGAP}

Freestanding nanogaps are useful. For example, to study single molecule conduction [12], nanogaps are made freestanding to facilitate molecule assembly procedure and to reduce current conduction through substrate. Here we show two ways to make freestanding nanogaps. The first method is to use $\mathrm{BrF}_{3}$ gas to dry etch the underneath sacrificial amorphous silicon (results in Fig. 8a). The

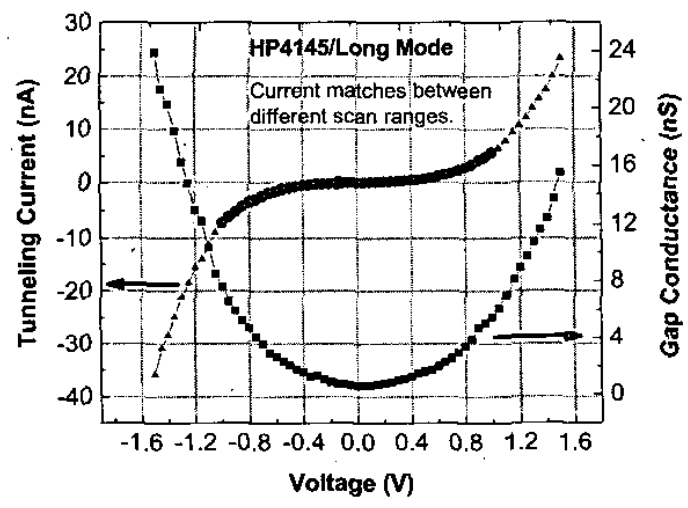

Fig. 7 Direct tunneling current and conductance measurement of nanometer gap. I-V from different voltage scan ranges overlap with each other. (a)

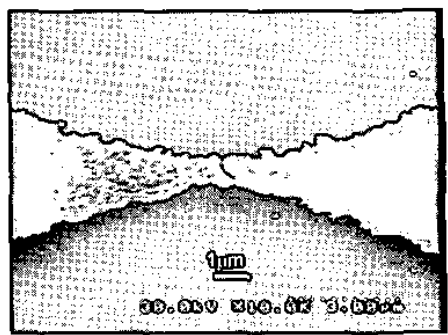

(b)

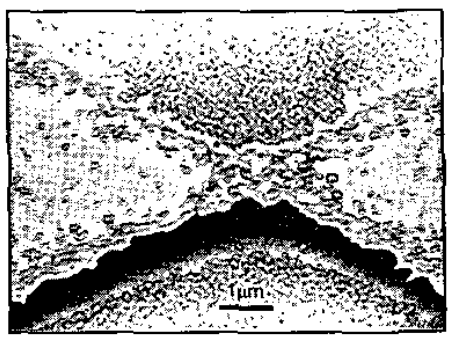

Fig.8 Freestanding Au electrodes anchored on amorphous silicon released by (a) $\mathrm{BrF}_{3}$ dry etching, (b) $\mathrm{SF}_{6}$ plasma etching.

drawback of this approach is the fast, but less controllable etching of $\mathrm{BrF}_{3}$ on silicon. Our observation shows that $\mathrm{Au}$ layer can stick to the oxide substrate when the undercut of amorphous silicon is larger than $10 \mu \mathrm{m}$. Electrostatic force generated from the oxide-trapped charge is believed to be the cause of stiction. The second method to make gaps freestanding is to use $\mathrm{SF}_{6}$ plasma etching (Fig. 8b).

Plasma etching of the silicon sacrificial layer is slower but controllable. The results show that this approach provides good silicon etching rate and the $\mathrm{Au}$ is not attacked throughout the process.

\section{SIZE CALIBRATION OF NANOGAP}

We develop a simple way to measure the freestanding nanogap distance. To do so, the freestanding length of the $\mathrm{Au}$ electrodes, i.e., anchor to anchor distance (L), is first measured ( $10 \mu \mathrm{m}$ for the example in Fig. 9). I-V curves are then measured at different temperatures increasing from $30{ }^{\circ} \mathrm{C}$ to $125^{\circ} \mathrm{C}$. Due to thermal expansion of $\mathrm{Au}$, which dominates over substrate thermal expansion, gap size decreases when temperature increases. When direct tunneling current shows up, the gap size is $\sim 5 \mathrm{~nm}\left(\mathrm{~g}_{0}\right)$. We can therefore calculate the initial room temperature gap size (g) according to thermal expansion principle (Eq. 1). Using this method, Fig. 9 data measures a gap distance of $14.6 \mathrm{~nm}$, compared to $15 \mathrm{~nm}$ measured from SEM picture.

\section{TUNABLE NANOGAP PRESSURE SENSOR}

As a preliminary demonstration to make nanogap distance tunable, we have built freestanding nanogap on top of a silicon membrane, which can be moved by pneumatic method. The gap distance is then tunable according to the membrane strain and the nanogap anchor 


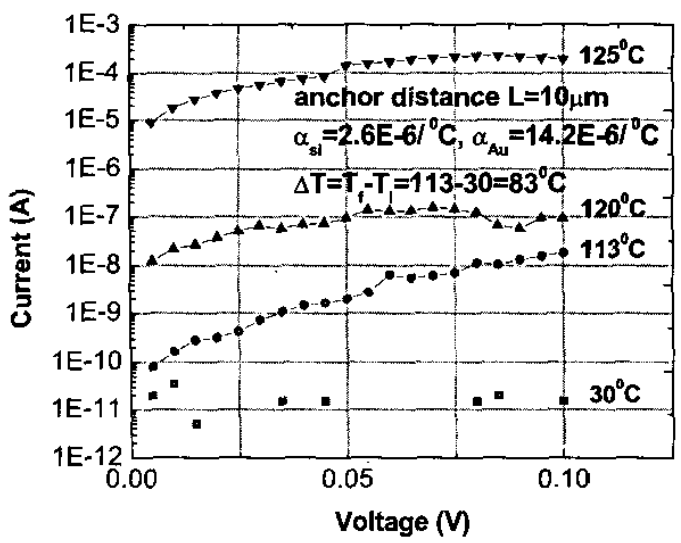

Fig. 9 Temperature method to calibrate freestanding nanogap size.

$$
\left\{\begin{array}{l}
\Delta g=\left(\alpha_{A u}-\alpha_{S i}\right) \cdot \Delta T \cdot L=9.6 \mathrm{~nm} \\
g=g_{0}+\Delta g=14.6 \mathrm{~nm}
\end{array}\right.
$$

-to-anchor distance. Results show that the tunneling is very sensitive to the gap distance, which is tuned by pressure (Fig. 10).

\section{CONCLUSION}

In this work, a feedback-controlled electromigration process is developed to create nanogaps $(<10 \mathrm{~nm})$ on thick Au films ( $>120 \mathrm{~nm})$. Compared with other methods, this approach requires no sophisticated equipment or procedure. It is highly compatible with standard CMOS or conventional MEMS fabrication process. Freestanding nanogaps can also be made using $\mathrm{SF}_{6}$ plasma free etching without inducing change of the gap distance. To calibrate the gap, a simple method based on thermal expansion principle is developed to measure the gap distance.

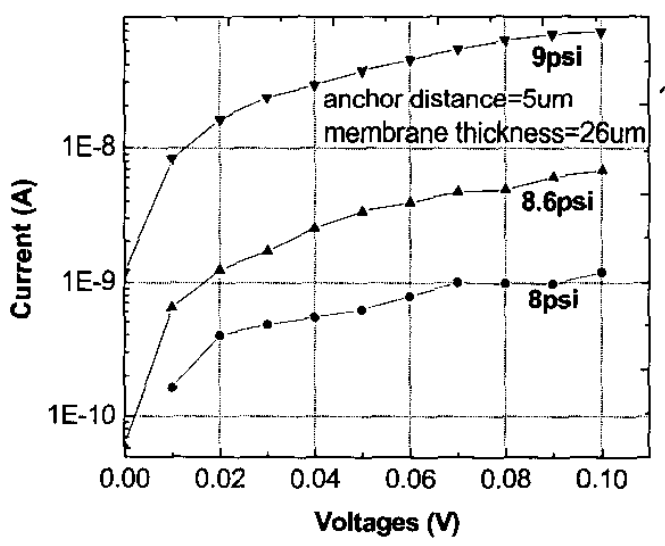

Fig.10 Demonstration of a nanogap pressure sensor using tunable gap. As the applied pressure changes by $1 \mathrm{psi}$, the tunneling current can change by two orders of magnitude.
Finally, the potential of using freestanding nanogap as a high resolution pressure sensor is demonstrated.

\section{ACKNOWLEDGMENTS}

This work is supported by the NSF ERC program at Caltech (Award No. EEC-9402726) and the NSF NIRT project (Award No. ECS-0103559).

\section{REFERENCES}

[1] H. Park, et al., "Fabrication of metallic electrodes with nanometer separation by electromigration", Appl. Phys. Lett. 75, 301 (1999).

[2] M.A. reed, et al., "Conductance of a Molecular junction", SCIENCE 278, 252, (1997).

[3] Y.V. Kervennic, et al., "Nanometer-spaced electrodes with calibrated separation", Appl. Phys. Lett. 80, 321, (2002).

[4] P.B. Fischer, et al., "10nm electron beam lithography and sub-50nm overlay using a modified scanning electron microscope", Appl. Phys. Lett. 62, 2989, (1993).

[5] C. Durkan, et al., "Analysis of the failure mechanisms of electrically stressed gold nanowires", J. Appl. Phys., 86, 1280, (1999).

[6] C.Y. Li, et al., "Electromigration-induced failure in passivated platinum-based metallizations-The dependence on temperature and current density", Appl. Phys. Lett. 61, 411, (1992).

[7] J.M. Poate, et al., "Thin Films-Interdiffusion and Reactions", chap.8, (1978).

[8] M. Austin, et al, "Fabrication of nanocontacts for molecular devices using nanoimprint lithography", J. Vac. Sci. technol. B 20, (2002).

[9] Robert Gomer, "Field Emission and Field Ionization", Chap.1, (1961).

[10] Gordon Francis, "Ionization. Phenomena in Gases", Chap.2, (1960).

[11] O.Yilmazoglu, et al., "Emission Behavior of Micromachined Vertical Field-Emitter with Cantilever Structure", Eurofe2001, Alicante(Spain), Nov.12-16, (2001).

[12] D. Porath, et al., "Direct measurement of electrical transport through DNA molecules", Nature, 403, 635, (2000). 